Article

\title{
Toxin $\zeta$ Reversible Induces Dormancy and Reduces the UDP- $N$-Acetylglucosamine Pool as One of the Protective Responses to Cope with Stress
}

\author{
Mariangela Tabone, Silvia Ayora and Juan C. Alonso * \\ Department of Microbial Biotechnology, Centro Nacional de Biotecnología, CNB-CSIC, C/Darwin 3, \\ Madrid 28049, Spain; E-Mails: mariangela.tabone@cnb.csic.es (M.T.); sayora@cnb.csic.es (S.A.) \\ * Author to whom correspondence should be addressed; E-Mail: jcalonso@cnb.csic.es; \\ Tel.: +34-91585-4546; Fax: +34-91585-4506.
}

Received: 18 June 2014; in revised form: 14 August 2014 / Accepted: 9 September 2014 /

Published: 18 September 2014

\begin{abstract}
Toxins of the $\zeta / \mathrm{PezT}$ family, found in the genome of major human pathogens, phosphorylate the peptidoglycan precursor uridine diphosphate- $N$-acetylglucosamine (UNAG) leading to unreactive UNAG-3P. Transient over-expression of a PezT variant impairs cell wall biosynthesis and triggers autolysis in Escherichia coli. Conversely, physiological levels of $\zeta$ reversibly induce dormancy produce a sub-fraction of membrane-compromised cells, and a minor subpopulation of Bacillus subtilis cells become tolerant of toxin action. We report here that purified $\zeta$ is a strong UNAG-dependent ATPase, being GTP a lower competitor. In vitro, $\zeta$ toxin phosphorylates a fraction of UNAG. In vivo, $\zeta$-mediated inactivation of UNAG by phosphorylation does not deplete the active UNAG pool, because expression of the toxin enhances the efficacy of genuine cell wall inhibitors (fosfomycin, vancomycin or ampicillin). Transient $\zeta$ expression together with fosfomycin treatment halt cell proliferation, but $\varepsilon_{2}$ antitoxin expression facilitates the exit of $\zeta$-induced dormancy, suggesting that there is sufficient UNAG for growth. We propose that $\zeta$ induces diverse cellular responses to cope with stress, being the reduction of the UNAG pool one among them. If the action of $\zeta$ is not inhibited, e.g., by de novo $\varepsilon_{2}$ antitoxin synthesis, the toxin markedly enhances the efficacy of antimicrobial treatment without massive autolysis in Firmicutes.
\end{abstract}

Keywords: toxin-antitoxin system; fosfomycin; vancomycin; ampicillin; bacterial persistence 


\section{Introduction}

Microbial type II toxin-antitoxin (TA) systems, which are stress regulators, are generally composed of two genes organized in an operon, encoding a toxin (T) and an antitoxin (A) protein [1-3]. RelE and $\zeta$ are the most abundant prokaryotic toxins in nature [4], with $\zeta$ been the most ubiquitous toxin in Firmicutes [5,6]. The plasmid- or chromosomal-encoded TA complexes ( $\zeta-\varepsilon$ or PezT-PezA) consist of two monomeric long-living $\zeta$ (PezT) toxins separated by a dimeric short-living $\varepsilon_{2}\left(\mathrm{PezA}_{2}\right)$ antitoxin, forming a $\zeta \varepsilon_{2} \zeta$ (PezTPezA ${ }_{2}$ PezT) inactive complex [7,8]. The toxins of the $\zeta$ family are highly conserved, $\zeta$ (286 amino acids long polypeptide) shares $42 \%$ of sequence identity with PezT (253 amino acids long). Both toxins have a very similar three-dimensional structure, and mutations in equivalent positions show similar phenotypes [7,8]. However, antitoxin $\varepsilon$ (90 amino acids long polypeptide) only shares $17 \%$ of sequence identity with the C-terminal region of antitoxin PezA (158 amino acids long). Despite this low sequence identity, the C-terminal region of PezA folds into a three helix bundle similar to that of $\varepsilon[7,8]$.

Both Firmicutes toxins, $\zeta$ and PezT might interact with $\mathrm{ATP}-\mathrm{Mg}^{2+}$ or $\mathrm{GTP}-\mathrm{Mg}^{2+}$, with uridine diphosphate- $N$-acetylglucosamine (UNAG) and with $\varepsilon_{2}$ (PezA) antitoxin [7,9]. These toxins, as part of the biological non-toxic heterotetrameric $\left(\zeta \varepsilon_{2} \zeta\right.$ or PezTPezA${ }_{2}$ PezT) complex, interact with UNAG [9]. However, in this inactive complex, the toxin cannot interact with ATP- $\mathrm{Mg}^{2+} / \mathrm{GTP}-\mathrm{Mg}^{2+}$, because the antitoxin helixes block the entry of the nucleotide cofactor into the binding pocket of the toxin through steric hindrance $[7,8,10]$. Toxin PezT phosphorylates the 3'-OH group, which is an essential precursor of bacterial cell wall biosynthesis, leading to phosphorylated, unreactive, UNAG-3P [9]. Toxin PezT was specific for UNAG, because it failed to phosphorylate uridine diphosphate- $N$-acetylgalactosamine [9]. Hence, $\zeta /$ PezT toxin blocks the first committed step in the peptidoglycan synthesis pathway, that is the transfer of an enolpyruvate residue from phosphoenolpyruvate to position 3 of UNAG [9].

Toxin $\zeta$, under various stress stimuli, can be activated as a result of $\varepsilon_{2}$ antitoxin degradation by stress-induced proteases or under conditions that prevent TA gene expression [8,11-13]. Such activation permits free wt $\zeta$ (or its short-living variant ( $\zeta \mathrm{Y} 83 \mathrm{C}$, half-life $\sim 28 \mathrm{~min}$ )) to trigger a heterogeneous set of protective responses, and the expression of $\sim 2 \%$ of total Escherichia coli or Bacillus subtilis genes, so that these toxins show a bacteriostatic, rather than a bactericidal

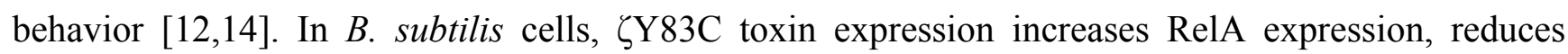
expression of lipid metabolism genes, and decreases the GTP pool without apparent alteration of the

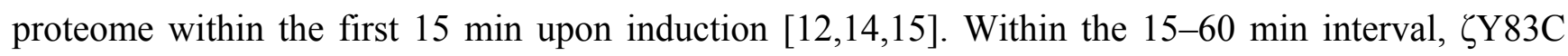
expression reduces the synthesis of macromolecules, decreases ATP levels, increases the alarmone guanosine (penta)tetraphosphate ([p] ppGpp) pool, and alters the membrane potential without affecting the SOS response [14,15]. Within 60-90 min, ¿Y83C expression decreases the pool of a novel metabolite, probably UNAG [14]. Finally, expression of $\zeta$ for 120-240 min leads to a fraction $(25 \%-35 \%)$ of the population stained with propidium iodide (PI), suggesting a membrane compromise; but subsequent expression of the $\varepsilon_{2}$ antitoxin facilitates the exit from the dormant state and a fraction of cells recover their membrane "alteration" [12,14,15]. A different effect is observed if B. subtilis cells express the wt $\zeta$ or $\zeta$ Y $83 \mathrm{C}$ toxin for longer periods of time ( $>960 \mathrm{~min}$ ). Here, the cells enter in a point of no return, and $\varepsilon_{2}$ antitoxin expression cannot reverse the dormant state $[12,16]$. Conversely, in E. coli cells PezT over-expression inhibits cell wall synthesis and triggers autolysis 
60 min after induction [9]. It is likely that toxin concentrations or time of exposure shows hormesis, complicating the understanding of $\zeta$ toxin mode of action.

\subsection{Aims of the Experiments}

Previously, it was been proposed that a PezT variant lacking the C-terminus (PezT $\Delta \mathrm{C} 242$ ) instigates a suicide program with subsequent autolysis [9], but $\zeta$ works as a checkpoint barrier inducing a reversible dormant state without massive cell lysis [14]. In the first outcome, PezT toxin may phosphorylate all the UNAG pool and by this way may provoke a cell membrane compromise and lysis, whereas $\zeta$ toxin acts on different cellular processes, not only cell wall synthesis. In order to discriminate between these two hypotheses, different experimental approaches were used in this report. First, $\zeta$ was purified, free of $\varepsilon_{2}$ antitoxin, to test whether ATP hydrolysis is coupled or not with the phosphate transfer to UNAG. In addition, we analyzed whether GTP can be used as a donor of the phosphate group. Second, we tested whether $\zeta$ expression depletes the UNAG pool or only reduces it. For that purpose, we used antimicrobials that inhibit cell proliferation by targeting cell wall synthesis with fosfomycin (Fos), vancomycin (Van), ampicillin (Amp) upon transient expression of the $\zeta$ Y83C toxin variant in $B$. subtilis cells. Third, we studied if upon specific reversal of $\zeta$-induced dormancy B. subtilis cell proliferation is recovered or there is cell lysis. To perform the latter study $\zeta$ was transiently expressed in the presence or absence of Fos, and then $\varepsilon_{2}$ antitoxin was induced. Finally, we tested whether $\zeta$ expression for long periods of time causes cell lysis in B. subtilis.

\section{Results and Discussion}

\subsection{Toxin $\zeta$ Hydrolyses ATP in the Presence of UNAG}

Recently, it was documented that in the presence of ATP- $\mathrm{Mg}^{2+}, \zeta$ toxin, in the presence of $\varepsilon_{2}$ antitoxin, phosphorylates the cell wall precursor UNAG by attaching a phosphoryl group to the 3'-hydroxyl group of the $N$-acetylglucosamine moiety to form UDP- $N$-acetylglucosamine-3'-phosphate (UNAG-3P) [9]. The low efficiency of this reaction, which was not stoichiometric, could be attributed to the presence of the antitoxin or because the phosphorylation reaction might be uncoupled from the ATPase reaction. In this work we have we tested whether $\zeta$ toxin purified by a novel protocol, free of antitoxin (as described in the Experimental section), is able to hydrolyze ATP in the presence or absence of UNAG. We have also tested whether the hydrolysis reaction is coupled with the phosphotransfer reaction and if GTP could work as a donor of inorganic phosphate $\left(\mathrm{P}_{\mathrm{i}}\right)$ for the transfer reaction. The products were separated from the substrate by thin-layer chromatography (TLC) and analyzed by autoradiography.

In the presence of UNAG, $\zeta$ toxin hydrolyzed $\left[\gamma^{32} \mathrm{P}\right]$-ATP, and we observed the accumulation of a radioactive product(s) which co-migrate with the front (data not shown). This was the expected position for the product of ATP hydrolysis, which is inorganic phosphate $\left[{ }^{32} \mathrm{Pi}\right]$. We do not know how $\left[\gamma^{32} \mathrm{P}\right]-\mathrm{UNAG}-3 \mathrm{P}$ should migrate under the conditions used, but one hypothesis is that it co-migrates with the TLC front under the experimental conditions used. To address this, we perform experiments with other TLC eluents in order to separate $\left[{ }^{32} \mathrm{P}_{\mathrm{i}}\right]$ from $\left[{ }^{32} \mathrm{P}\right]-U N A G-\mathrm{P}$, but we failed to have a significant separation of both of them under the conditions used (data not shown). Alternatively, 
the reaction is uncoupled and $\left[\gamma^{32} \mathrm{P}\right]-\mathrm{UNAG}-\mathrm{P}$ accounts only to a minor fraction of the radiolabelled product.

To quantify the rate of ATP hydrolysis the experiments were performed using $\left[\alpha^{32} \mathrm{P}\right]-\mathrm{ATP}$. If $\zeta$ hydrolyses $\left[\alpha^{32} \mathrm{P}\right]$-ATP it should be converted onto $\left[\alpha^{32} \mathrm{P}\right]$-ADP that runs faster than $\left[\alpha^{32} \mathrm{P}\right]$-ATP in a TLC. The commercial radioactive $\left[\alpha^{32} \mathrm{P}\right]$-ATP contains $6 \%-10 \%$ of $\left[\alpha^{32} \mathrm{P}\right]$-ADP [17], hence this is our background level, and this was used in the TLC as an internal control that marked the respective running positions of substrates and products. In the absence of $\zeta$ toxin no $\left[\alpha^{32} \mathrm{P}\right]$-ATP hydrolysis was observed (Figure 1, lane 10$)$. Purified $\zeta$ toxin $(0.5 \mu \mathrm{M})$ did not hydrolyze $\left[\alpha^{32} \mathrm{P}\right]$-ATP $(0.5 \mathrm{mM})$ in the absence of UNAG, but $\zeta$ toxin hydrolyzed $>85 \%$ of the ATP substrate upon addition of UNAG $(2 \mathrm{mM})$, in a $60 \mathrm{~min}$ reaction (Figure 1, lanes 1 and 2 ).

Figure 1. Uridine diphosphate- $N$-acetylglucosamine (UNAG)-dependent $\zeta$ hydrolysis of ATP is poorly competed by GTP. Samples containing $2 \mathrm{mM}$ UNAG and increasing concentrations of ATP $\left(0.5,5\right.$ and $10 \mathrm{mM}$ (with a fixed concentration of $\alpha^{32} \mathrm{P}-\mathrm{ATP}$, $10 \mathrm{nM})$, lanes 2-4) or $2 \mathrm{mM} \mathrm{UNAG}$ and a fixed concentration of ATP $\left(0.5 \mathrm{mM}\left[\alpha^{32} \mathrm{P}-\mathrm{ATP}\right.\right.$, $10 \mathrm{nM}])$ and increasing GTP concentrations $(1.25,2.5,5,7.5$ and $10 \mathrm{mM}$, lanes 5-9) were incubated with $0.5 \mu \mathrm{M} \zeta$ toxin for $30 \mathrm{~min}$ at $30{ }^{\circ} \mathrm{C}$ in buffer B. ATP hydrolysis was analyzed by thin-layer chromatography (TLC) performed on polyethyleneimine-cellulose plates with $0.85 \mathrm{M} \mathrm{KH}_{2} \mathrm{PO}_{4}$ (pH 3.4) as the mobile phase, followed by autoradiography.

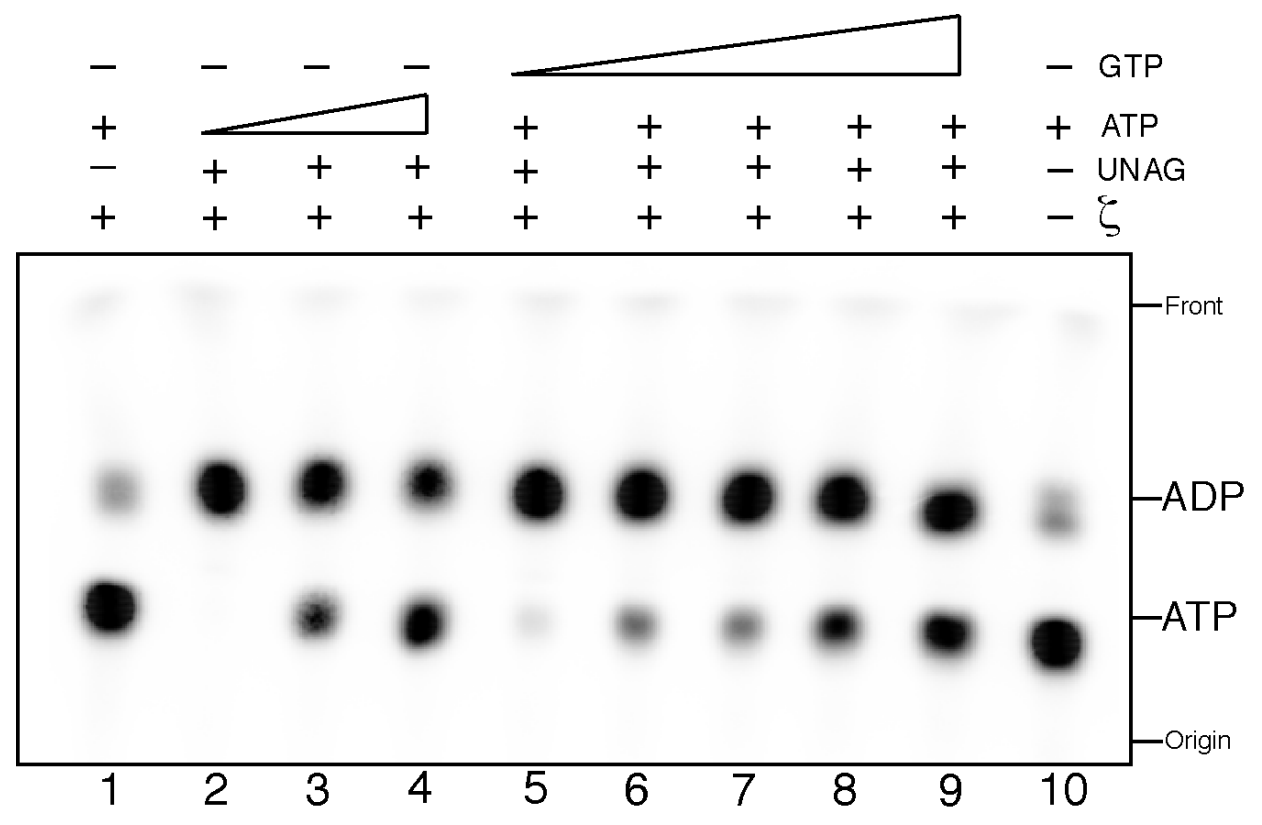

When saturating ATP concentrations were used, also a large fraction of the ATP was hydrolyzed (Figure 1, lanes 3 and 4). At ATP:UNAG ratios of $2.5: 1$ or 5:1 70\% and $\sim 40 \%$ of radiolabelled $\left[\alpha^{32} \mathrm{P}\right]$-ATP was converted onto $\left[\alpha^{32} \mathrm{P}\right]$-ADP, respectively, in a $60 \mathrm{~min}$ reaction (Figure 1 , lanes 3 and 4). Since the conversion of ATP onto ADP was significantly higher than if each $\mathrm{P}_{\mathrm{i}}$ generated was transferred to the 3'-OH group of the sugar moiety of UNAG, we tentatively assume that the reaction is not tightly coupled. Previously, it was shown that $\zeta$ toxin, in the presence of ATP- $\mathrm{Mg}^{2+}$ and the $\varepsilon_{2}$ antitoxin, attaches a phosphoryl group to the 3'-hydroxyl group of the $N$-acetylglucosamine moiety to form UDP- $N$-acetylglucosamine-3'-phosphate (UNAG-3P) [9]. Hence we assume that $\zeta$ toxin alone 
(free of $\varepsilon_{2}$ antitoxin) would also transfer the $\mathrm{P}_{\mathrm{i}}$ to form UNAG-3P, but it remains to be shown whether the 3'-OH group of the sugar moiety of UNAG is the only target of the phosphorylation reaction.

To verify the structurally suggested preference for ATP vs. GTP (as reported for other phosphotransferases) as substrate, we performed competition experiments. Here, $\zeta$ toxin was incubated with increasing ATP or GTP concentrations containing a fixed amount of ATP (0.5 mM ATP containing $\left[\alpha-{ }^{32} \mathrm{P}\right]$-ATP $\left.10 \mathrm{nM}\right)$ and UNAG $(2 \mathrm{mM})$. Toxin $\zeta$ can hydrolyze ATP with similar efficiency in the presence of a 2.5-fold excess of GTP (Figure 1, lane 5). GTP:ATP ratios of 5:1 to 10:1, marginally decreased $\zeta$-mediated $\left[\alpha^{32} \mathrm{P}\right]$-ATP hydrolysis (Figure 1, lanes 6 and 7). At GTP:ATP ratios of $15: 1$ and $20.5: 1, \sim 37$ and $\sim 46 \%$ of $\left[\alpha^{32} \mathrm{P}\right]$-ATP was not converted to [ $\left.\alpha^{32} \mathrm{P}\right]$-ADP, respectively (Figure 1, lanes 8 and 9). It is likely therefore that: (I) $\zeta$ toxin is preferentially a UNAG-dependent ATPase; (II) the phosphotransfer reaction might not simply be a one-step coupled reaction; and (III) $\zeta$ toxin preferentially hydrolyzed ATP- $\mathrm{Mg}^{2+}$ over GTP- $\mathrm{Mg}^{2+}$ in the presence of UNAG.

\subsection{Toxin $\zeta$ Phosphorylates a Fraction of UNAG In Vitro}

In the previous section was shown that $\zeta$ requires the presence of UNAG to hydrolyze ATP, suggesting that UNAG or UNAG-3P might stimulate $\zeta$-mediated ATP hydrolysis. To further analyze this, UNAG and ATP were incubated in the absence or presence $\zeta$ and the products of the reaction were analyzed by mass spectrometry as described in the Experimental section.

In a mock reaction, lacking the $\zeta$ toxin, phosphorylated UNAG was not observed (compare Figure 2A,B). In all conditions the nucleotide (or compound) and its Na-bound (e.g., $\mathrm{M}-\mathrm{H}^{+}$ [ATP, 506.06 peak], the $\mathrm{M}-2 \mathrm{H}^{+}-\mathrm{Na}^{+}$[ATP-Na, 528.05 peak] or even $\mathrm{M}-3 \mathrm{H}^{+}-2 \mathrm{Na}^{+}$forms (due to the contribution of the buffer used) were detected (Figure 2A). In the presence of $\zeta$ toxin, a massive degradation of the ATP pool (506.06 Da peak, expected 507.18 Da) with subsequent accumulation of ADP (426.06 Da peak, expected 427.20 Da) was observed. However, the proportion of UNAG (606.15 Da UNAG peak, expected 607.35 Da) converted into a 687.35 Da product (UNAG-3P) with a small peak at $686.12 \mathrm{Da}$ (UNAG-3P-Na $)$ was detectable but poor (Figure 2). A parallel TLC analysis of this reaction revealed that $\zeta$ toxin hydrolyzed more than $95 \%$ of the ATP substrate (data not shown).

As revealed in Figure 2B, in the presence of $\zeta$ toxin traces of the ATP pool and a reduced fraction of UNAG were detected by mass spectrometry, when compared to the absence of $\zeta$ toxin (Figure 2A). Since traces of ATP and a significant fraction of UNAG remained after 30 min reaction, it was assumed, as done in the previous section (Figure 1), that only a fraction of the $\mathrm{P}_{\mathrm{i}}$ was transferred to UNAG to generate UNAG-3P (Figure $2 \mathrm{~B}$ ). Alternatively, $\zeta$ toxin phosphorylates UNAG in a coupled reaction, but under the conditions used we are loosing a fraction of the accumulated inactive UNAG-3P product. 
Figure 2. $\zeta$ toxin phosphorylates in vitro a fraction of UNAG. Samples containing $2 \mathrm{mM}$ UNAG and $0.5 \mathrm{mM}$ ATP were incubated in the absence $(\mathbf{A})$ or presence $(\mathbf{B})$ of $\zeta$ toxin $(0.5 \mu \mathrm{M})$ for $60 \mathrm{~min}$ at $30{ }^{\circ} \mathrm{C}$ in buffer $\mathrm{B}$. The reaction products were analyzed by mass spectroscopy. The peaks corresponding to relevant products (ATP, ADP, UNAG and UNAG-3P) are indicated. The intensity $\left(\times 10^{4}\right)$ was expressed in arbitrary units (a.u.). In panel $\mathrm{B}$, the 675 to $740 \mathrm{~m} / \mathrm{z}$ section is enlarged in the insert.

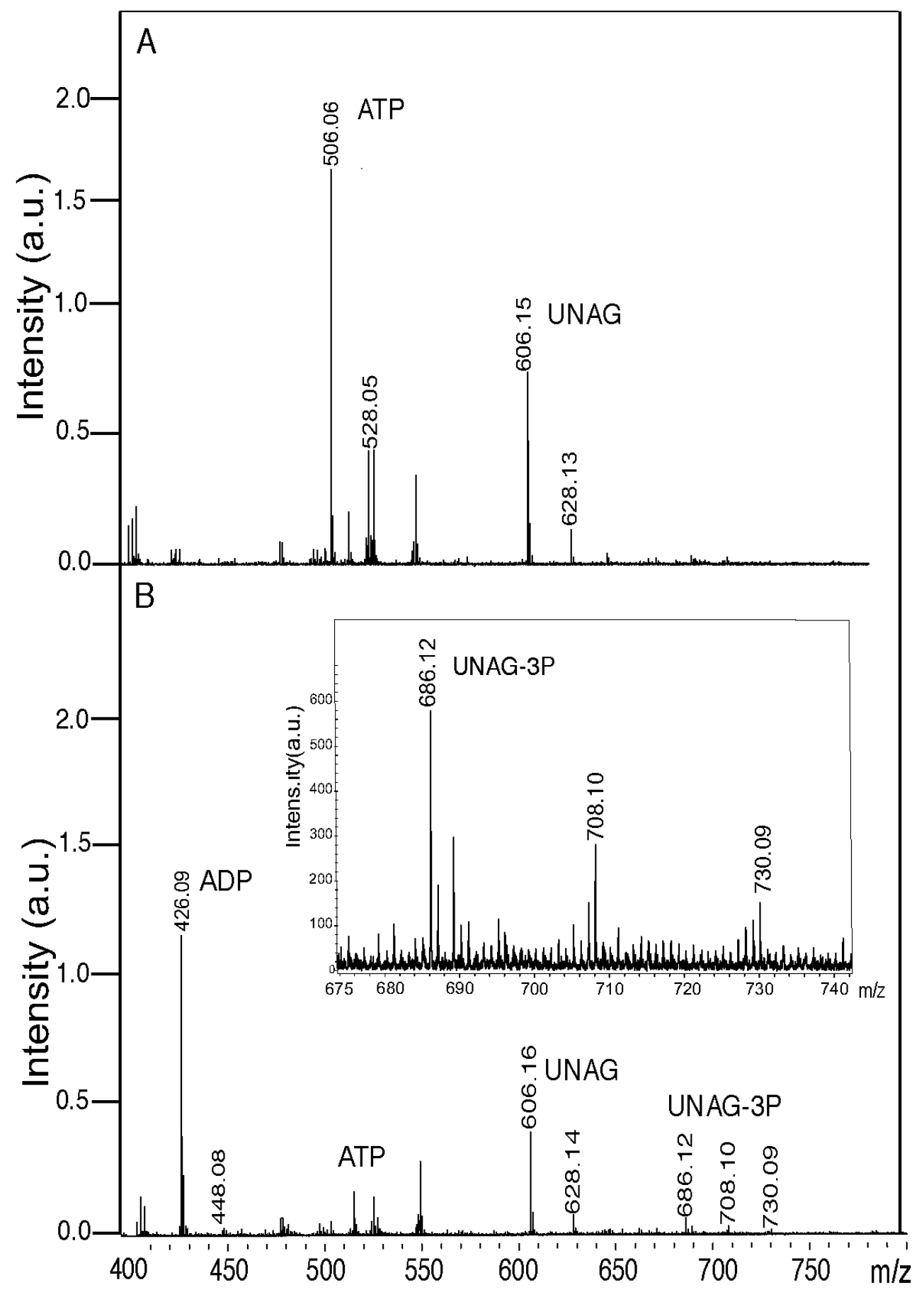




\subsection{Toxin \Y83C Leaves Significant Amounts of UNAG In Vivo}

In vitro assays showed that $\zeta$ is a poor kinase, phosphorylating only a fraction of the UNAG present. However, these assays might not extrapolate to the in vivo situation. It could be that in vivo there is(are) some unknown factor(s) that may stimulate the phosphotransfer reaction. To test whether $\zeta$ toxin reduces or depletes the UNAG pool in vivo, and by this way it reduces or blocks the biosynthetic pathway of peptidoglycans different types of antimicrobials, which act at different levels of the two stage process of cell wall biosynthesis, were used.

It has been established that the first step of peptidoglycan (murein) biosynthesis is a cytosolic stage that consists in the transfer of an enolpyruvate moiety from phosphoenolpyruvate (PEP) to the C3 position of $\mathrm{N}$-acetylglucosamine moiety of UNAG, a reaction catalyzed by two MurA enzymes in Firmicutes (the ubiquitous MurA [MurAA or MurA1] and MurAB [or MurA2] [18]), to yield enolpyruvyl-UDP- $N$-acetylglucosamine (EP-UNAG). The synthesis of these murein precursors can be

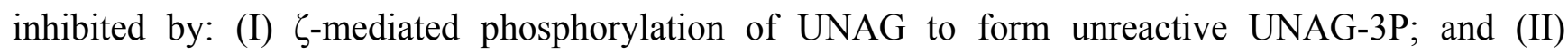
a naturally occurring PEP analogue, Fosfomycin (Fos), which binds covalently to the active site of MurAA (or MurAB). Fos, in the presence of the UNAG substrate inhibits cell wall biosynthesis by blocking the accumulation of EP-UNAG [19,20]. EP-UNAG is transformed by a series of cytosolic steps, which results in the formation of the UDP- $N$-acetyl-muramic acid-pentapeptide that is translocated to the membrane acceptor and through different steps the $N$-acetylglucosamine $/ N$-acetylmuramic acid-pentapeptide is covalently linked to the lipid carrier molecule undecaprenol via a pyrophosphate ester bridge (Lipid II). Vancomycin (Van) prevents incorporation of $N$-acetylglucosamine and $\mathrm{N}$-acetylmuramic acid-peptapeptide subunits into the peptidoglycan matrix, and interferes with the Lipid II cycle [21]. Then, the Lipid II building block is used as the substrate for the polymerization reaction, consisting of the assembly into glycan chains by transglycosilation and peptide cross-linking of the pentapeptide moiety by transpeptidation, reactions catalyzed by Classes A or B penicillin-binding proteins [22-24]. Amp acts as an irreversible inhibitor of these transpeptidases [25,26].

We reasoned that if $\zeta$ toxin depletes the UNAG pool, cells will become tolerant to antimicrobials that act downstream of toxin action, leading to non-inheritable drug tolerance (persistence). To test this hypothesis, $\zeta$ toxin induced cells were exposed to Fos, Van or Amp and the antimicrobial drug tolerance was analyzed. These antimicrobials, in addition of inhibiting cell wall synthesis as explained above, induce two regulatory systems to cope with stress in Firmicutes: the extracytoplasmic function $(\mathrm{ECF}) \sigma$ factors and/or cell envelope stress-sensing two component systems, which are linked to other stress responses [27]. Van, which is the strongest cell envelope-perturbing agent, induces the synthesis of a large number of genes controlled by specific $\sigma$ factors $\left(\sigma^{\mathrm{V}}, \sigma^{\mathrm{M}}, \sigma^{\mathrm{W}}\right.$ and $\left.\sigma^{\mathrm{Y}}\right)$ and two components systems (e.g., LiaRS), Fos alters the expression of genes controlled by $\sigma^{\mathrm{M}}$ and $\sigma^{\mathrm{W}}$; and Amp alters the expression of genes through cell envelope stress-sensing two component systems (e.g., BlaRI, MecRI) [27]. If the three antimicrobials show a similar behavior upon $\zeta$ toxin we can omit any specific contribution of cell envelop stress.

To perform these experiments we took advantage of the short-lived toxin variant, ¿Y83C (half-life $\sim 28 \mathrm{~min}$ ) under control of a Xyl inducible promoter ( $x y l R P_{\mathrm{XylA}} \zeta \mathrm{Y} 83 \mathrm{C}$ expression cassette) (Table 1). The level of toxin in non-induced BG689 cells is too low ( $<10 \zeta Y 83 \mathrm{C}$ monomers/colony forming units, CFUs) to measurably alter the growth rate in minimal medium S7 (MMS7) [12]. 
Previously it was observed that induction of the $x y l R P_{\mathrm{XylA}} \zeta \mathrm{Y} 83 \mathrm{C}$ cassette (BG689 cells), by addition of $0.5 \% \mathrm{Xyl}$, increased $\zeta \mathrm{Y} 83 \mathrm{C}$ levels to a plateau with a toxin concentration of $\sim 300$ toxin monomers/CFUs at $\sim 10 \mathrm{~min}$, and the steady-state level of the toxin remained for at least $240 \mathrm{~min}$ [12]. When exponentially growing BG689 cells, growing in MMS7, reached moderate-density $\left(\sim 5 \times 10^{7}\right.$ cells $/ \mathrm{mL}$ ) xylose (Xyl) $0.5 \%$ was added to express $\zeta Y 83 \mathrm{C}$ toxin as described Materials and Methods [14,15]. As already observed, transient exposure to sub-physiological concentrations of free ¿Y83C induced dormancy and produced a typical biphasic survival curve, with a sub-fraction of cells $\left(1-5 \times 10^{-5}\right.$ cells) non-inheritable tolerant to toxin action upon $120 \mathrm{~min}$ of exposure (Figure 3) $[14,15]$.

Table 1. Bacterial strains used.

\begin{tabular}{|c|c|c|}
\hline Strains & Relevant genotype & Reference \\
\hline${\mathrm{BG} 687^{\mathrm{a}}}^{\mathrm{a}}$ & $+x y l R, P_{x y l A}, c a t$ & [12] \\
\hline BG689 ${ }^{\text {a }}$ & $+x y l R, P_{x y l A} \zeta \mathrm{Y} 83 \mathrm{C}, \mathrm{cat}$ & [12] \\
\hline $\mathrm{BG} 1127^{\mathrm{a}}$ & $+l a c I, P_{h s p}, s p c,\left[\mathrm{pCB} 799, x y l R, P_{x y l A} \varepsilon, c a t\right]$ & [12] \\
\hline BG1125 a,b & $+l a c I, P_{h s p} \zeta, s p c,\left[\mathrm{pCB} 799, x y l R, P_{x y l A} \varepsilon, c a t\right]$ & {$[12]$} \\
\hline BL21(DE3) ${ }^{\mathrm{c}}$ & $+\left[\mathrm{pCB} 920, P_{\mathrm{T} 7} \zeta\right.$ gene, $P_{\omega} \omega$ and $\varepsilon$ genes, $\left.b l a\right]$ & This work \\
\hline \multicolumn{3}{|c|}{ 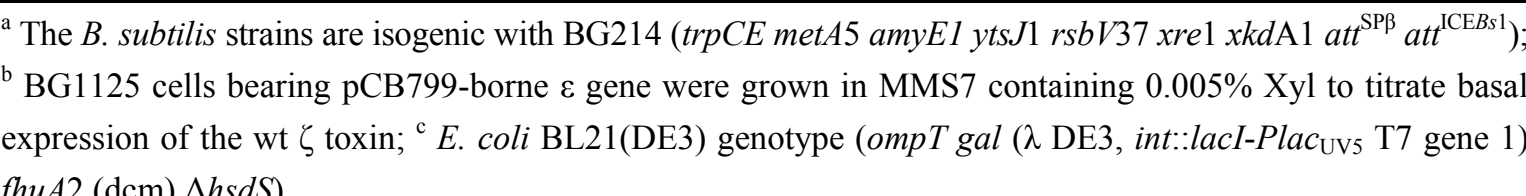 } \\
\hline
\end{tabular}

When exponentially growing BG689 cells $\left(\sim 5 \times 10^{7}\right.$ cells/mL in MMS7) were transiently exposed to Fos or Amp (at twice the minimal inhibitory concentration [MIC]) or to Van (at 4 times the MIC) for $120 \mathrm{~min}$, these bactericidal antimicrobials caused a cessation of cell proliferation, and rendered a fraction of 0.8 to $5 \times 10^{-3}$ phenotypic tolerants (Figure 3). This multidrug tolerance of the isogenic population of antimicrobial sensitive cells is termed bacterial persistence [28-30].

To test whether toxin action depletes the UNAG pool and this leads to enhanced antimicrobial persistence when cells are treated with cell wall inhibitors moderate-density exponentially growing BG689 cells were treated with Xyl (to induce $\zeta Y 83 \mathrm{C}$ toxin expression) and transiently exposed to the antimicrobial. Experiments are showed when the two compounds (i.e., Xyl and the antimicrobial) were added simultaneously, but the same results were obtained when first cells were exposed to toxin action for $30 \mathrm{~min}$, and later the antimicrobial was added. Toxin $\zeta Y 83 \mathrm{C}$ expression and Fos, Van or Amp treatment additively decreased the rate of cell survivals to 0.9 to $2 \times 10^{-6}$ (Figure 3). Since the three antimicrobial show a similar outcome in the presence of free $\zeta Y 83 \mathrm{C}$ toxin, it is unlike that the enhanced sensitivity could be attributed to a perturbation of cell envelop stress (see above). It is likely that: (I) $\zeta$ toxin expression only decreases the UNAG pool, and Fos, Van or Amp use the remaining fraction to further inhibit the plating of toxin tolerant cells; and (II) $\zeta$ toxin expression additively enhances the efficacy of the antimicrobials rather than making cells insensitive to Fos, Van or Amp treatment. Alternatively, transient expression of $\zeta$ toxin, by inactivating the UNAG substrate, inhibits the action of the MurA-MurF enzymes, and this inhibition leads to loss of cell shape and integrity, with cells becoming more prone to autolysis, when they are additionally treated with any of these antimicrobial. 
Figure 3. Cell wall inhibitors and $\zeta$ toxin expression show an additive effect. BG689 cells containing the short living $\zeta$ variant ( $\zeta$ Y $83 \mathrm{C}$ ) gene were grown to $\sim 5 \times 10^{7} \mathrm{cell} / \mathrm{mL}$ in MMS7. Then Xyl ( $0.5 \%$ to induce expression of the $\zeta Y 83 \mathrm{C}$ toxin) or an antimicrobial or both was added and the culture was incubated for $120 \mathrm{~min}$. Cells were washed and appropriate dilutions were plated to count the survivals. The results are the average of at least four independent experiments and are within a 10\% standard error.

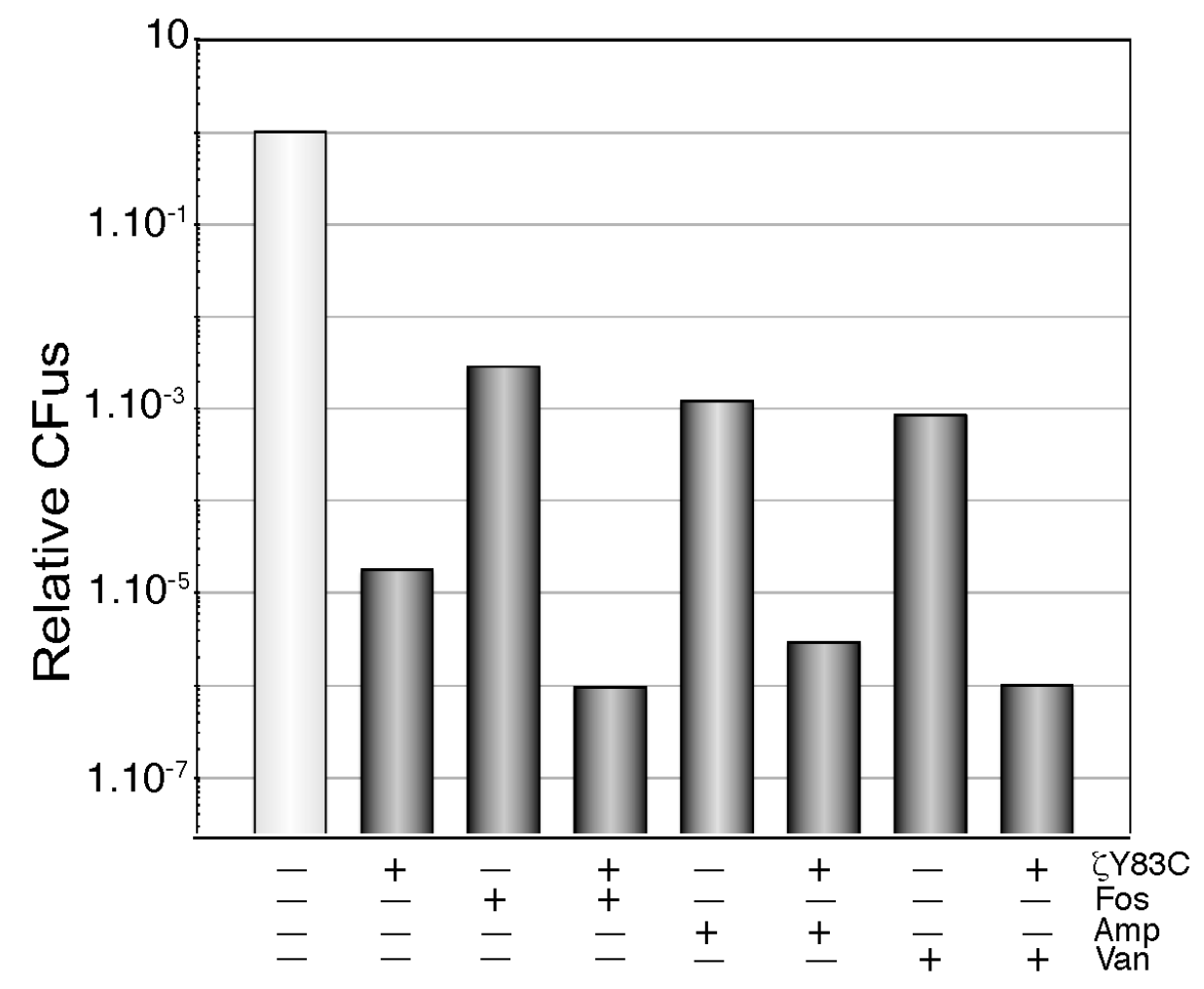

\subsection{Prolonged Action of Toxin \Y83C Does not Induce Massive Cell Lysis}

To test the latter hypothesis were analyzed if prolonged expression of sub-physiological concentrations of $\zeta Y 83 \mathrm{C}$ (360 or $480 \mathrm{~min}$ ) might exhaust the metabolite pool and BG689 cells might reach the limits of their "capacity" to exit the dormant state, and they cannot be longer rescued from the "point of no return". As already observed, after $16 \mathrm{~h}$ of $\zeta \mathrm{Y} 83 \mathrm{C}$ toxin expression the survival rate drops to $2-8 \times 10^{-7}[16]$.

BG689 cells were grown up to optical density $\left(\mathrm{OD}_{600}\right)$ of 0.2 in MMS7 and divided into two aliquots. To one aliquot inducer $(0.5 \% \mathrm{Xyl})$ was added (time zero) and samples were collected at different times (120, 240, 360 and $480 \mathrm{~min}$ after induction) (Figure 4). In the presence of Xyl, the increment in $\mathrm{OD}_{600}$ halted after one doubling time, and remained constant during the $480 \mathrm{~min}$ interval (Figure 4A). At these time points, samples were withdrawn, and the cells were stained with membrane-permeant SYTO 9 (green fluorescence) and membrane-impermeant PI (red fluorescence), appropriate dilutions were plated in LB plates and the survival rate was measured (Figure 4B). Transient expression of $\zeta Y 83 \mathrm{C}$ toxin for $120 \mathrm{~min}$ or longer periods of time induced dormancy, and a similar sub-fraction of $2-6 \times 10^{-5}$ survivals became tolerant of toxin action (Figure 4B). Within the first $360 \mathrm{~min}$ of $\zeta Y 83 \mathrm{C}$ toxin expression, the proportion of PI permeable cells slightly increased ( $\sim 1.2$-fold). At later times $(480 \mathrm{~min})$ the proportion of PI stained cells increased $\sim 2$-fold $(\sim 65 \%$ of total 
cells stained with PI) with respect to the proportion of cells stained with PI after 120 min of toxin expression (Figure 4B). It is likely that prolonged $\zeta$ Y $83 \mathrm{C}$ expression might not induce massive cell lysis.

Figure 4. Effect of prolonged action of toxin $\zeta Y 83 \mathrm{C}$ on membrane permeability and cell survival. (A) Growth curve of BG689 cells containing $\zeta Y 83 \mathrm{C}$ gene. Cells were grown to $\sim 2.5 \times 10^{7} \mathrm{cell} / \mathrm{mL}$ in MMS7 $\left(\mathrm{OD}_{600}=0.2\right)$, to half of the culture Xyl $(0.5 \%)$ was added to induce $\zeta Y 83 \mathrm{C}$ expression (time zero) and $\mathrm{OD}_{600}$ was followed over time in the two cultures. The arrow denotes the time of Xyl (0.5\%) addition; (B) Aliquots of the cultures were taken at time zero, 120, 360 and 480 min after Xyl addition (denoted as + ). The cells were fixed, stained with SYTO 9 and PI, and analyzed by fluorescence microscopy (black lane), at the same time, cells were washed and appropriate dilutions were plated in LB plates to count the survivals. The numbers of relative CFUs (black bars) at the indicated times after $\zeta$ Y $83 \mathrm{C}$ toxin expression are relative to the non-induced control at the same time (denoted as -) taken as 1 (grey bar). Error bars show 95\% confidence intervals of more than three independent experiments.
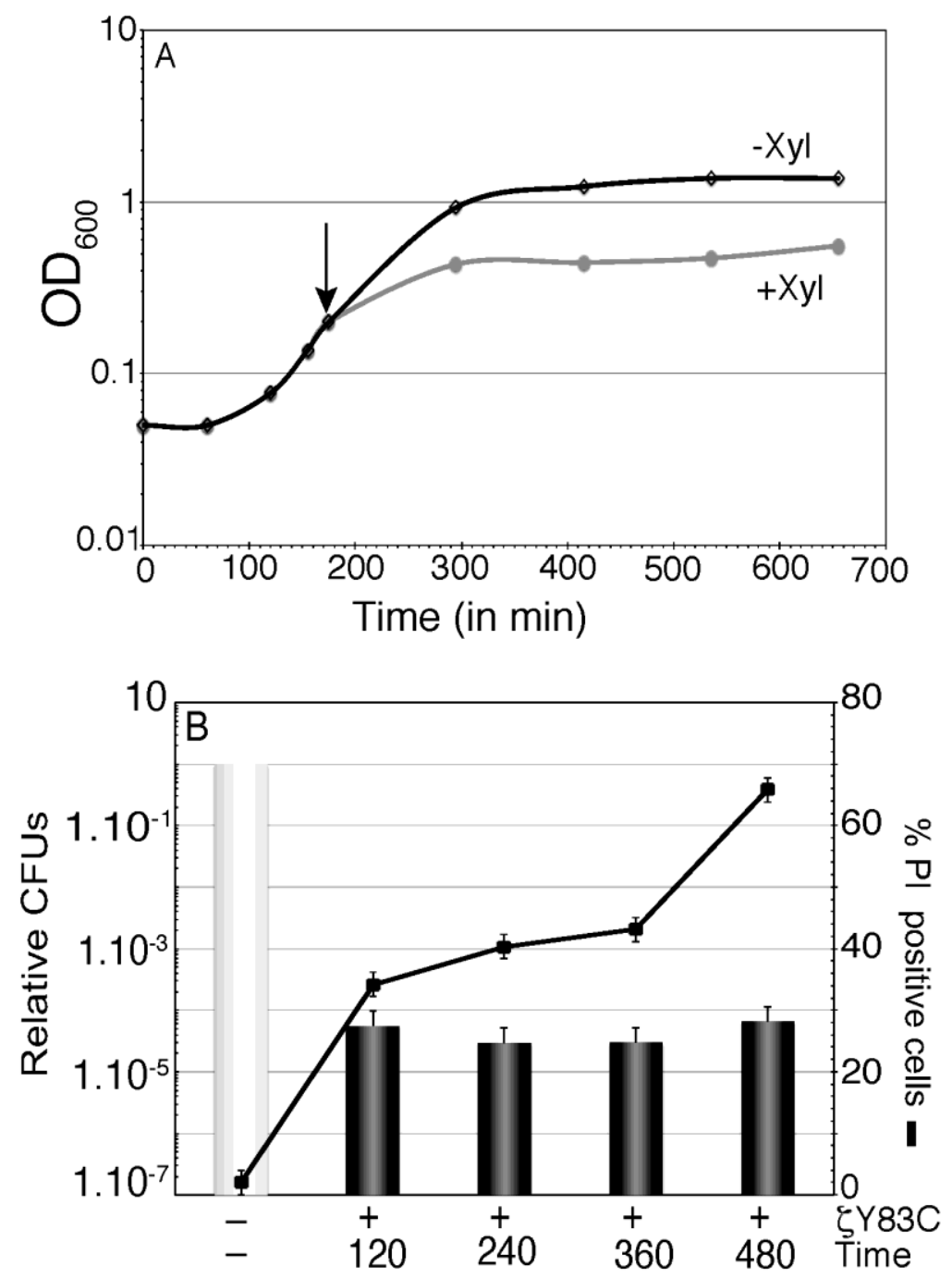
When similar experiments were performed in E. coli cells a different outcome is observed depending of the growth medium rather than the speed of growth [9,31]: In LB medium (doubling time $\sim 28 \mathrm{~min}$ ), $60 \mathrm{~min}$ after overexpression of PezT $\Delta \mathrm{C} 242$, cells underwent a massive death with few surviving cells showing an ovoid morphology [9]; in supplemented M9 medium (doubling time 33 min), $\zeta$ toxin over-expression induced dormancy, and $60 \mathrm{~min}$ after induction massive filamentation, with subsequent growth recovery at later times was observed [31]. At present, the source of these discrepancies remains unknown.

\subsection{Toxin $\zeta$ Reversibly Induces a Halt in Cell Proliferation}

Previously, it was shown that transient exposure $(120 \mathrm{~min})$ to physiological concentrations of free wt $\zeta$ reversibly induces dormancy, produces a sub-fraction of membrane-compromised cells to be stained with PI (25\%-35\% of total cells), and a minor subpopulation of B. subtilis cells become tolerant of toxin action. Subsequent expression of the $\varepsilon_{2}$ antitoxin facilitates the exit from the dormant state and a fraction of membrane compromised cells recover their alteration of the membrane potential [14].

Toxin $\zeta$ expression inactivated a fraction of UNAG and Fos used the remaining fraction of unphosphorylated UNAG to further inhibit cell proliferation (see Figure 3 ). If $\zeta$ toxin expression only reduces the UNAG pool and/or UNAG-P poisons the exit from the dormant state, expression of $\varepsilon_{2}$ antitoxin might lead to different outcomes in the presence of Fos: First, if $\zeta$ toxin expression and Fos addition deplete the UNAG pool, cells should not be recovered by antitoxin expression and cell wall biosynthesis can be rescued only after de novo synthesis of UNAG or UNAG-3P has to be re-activated by an unknown pathway. Second, the concert action of both $\zeta$ toxin expression and Fos addition triggers cell lysis by unbalancing the control of peptidoglycan biosynthesis. Finally, if $\zeta$ toxin expression decreases a fraction of the UNAG pool, without depleting it, $\varepsilon_{2}$ antitoxin expression may reverse toxin action and the remaining UNAG is used to rescue cell proliferation. To discriminate between all these possible outcomes, cell survival and the proportion of PI stained cells were analyzed after $\varepsilon_{2}$ antitoxin expression.

When exponentially growing BG1125 cells (Table 1$)$ reached a moderate-density $\left(\sim 5 \times 10^{7}\right.$ cells $/ \mathrm{mL}$ in MMS7) $1 \mathrm{mM}$ IPTG was added to induce $\zeta$ toxin expression. As already observed, expression of nearly physiological wt $\zeta$ toxin concentrations for 120 min induced dormancy, a fraction of the cell population $(\sim 30 \%)$ was stained with PI, and $\sim 1 \times 10^{-5}$ survivals tolerant to toxin action were obtained (Table 2). Subsequent expression of the $\varepsilon_{2}$ antitoxin, after $120 \mathrm{~min}$ of IPTG addition, by Xyl (0.5\%) addition, led to exit of the dormant state with CFUs increasing $\sim 5000$-fold, and only a small fraction $(\sim 10 \%)$ of cells were stained with PI (Table 2$)$ [14,15]. Alternatively, resumption of cell growth might dilute the relative proportion of PI stained cells up to $10 \%$ of total cells.

In the absence of IPTG, addition of Fos to moderate-density exponentially growing BG1125 cells for 120 min reduced plating efficiency of the cells to levels similar to the ones observed in the BG689 background $\left(\sim 3 \times 10^{-3}\right.$ survivals), and as expected for a bactericidal antimicrobial the proportion of cells permeable to PI increased ( $\sim 70 \%$ of total cells) (Table 2$)$. Expression of the $\varepsilon_{2}$ antitoxin under these conditions did nor alter the observed outcome ( $\sim 70 \%$ of total cells stained with PI, and $\sim 3 \times 10^{-3}$ survivals). When moderate-density exponentially growing BG1125 cells were exposed to toxin 
(by addition of $1 \mathrm{mM} \mathrm{IPTG)} \mathrm{and} \mathrm{Fos} \mathrm{action,} \mathrm{the} \mathrm{toxin} \mathrm{markedly} \mathrm{enhanced} \mathrm{the} \mathrm{efficacy} \mathrm{of} \mathrm{Fos} \mathrm{treatment,}$ but no additive effect on membrane permeation to PI was observed (Table 2). Then, transient expression of the $\varepsilon_{2}$ antitoxin led to partial exit of the dormant state, which increased $>2000$-fold CFUs, but it failed to decrease the proportion of PI permeable cells (Table 2). It is likely that: (I) toxin expression enhances Fos sensitivity, and such effect does not seem to correlate with increased rate of cell lysis; (II) neither toxin expression nor Fos addition deplete the UNAG pool, because $\varepsilon_{2}$ antitoxin expression facilitates the exit of the dormant state of a large fraction of cells; and (III) unregulated levels of murein synthesis, by transient toxin expression and Fos addition, do not induce massive cell autolysis. We propose that $\zeta$ toxin and Fos decrease the UNAG pool, but when $\varepsilon_{2}$ antitoxin expression halts $\zeta$ toxin activity, by forming an inactive complex $\left(\zeta \varepsilon_{2} \zeta\right)$, the remaining active fraction of UNAG is processed by MurA enzymes and cells exit the $\zeta$-induced dormant state with resumption of growth as expected for a toxin that is bacteriostatic in nature.

Table 2. Additive effect of $\zeta$ toxin on Fos action and $\varepsilon_{2}$ reversion of $\zeta$-induced dormancy.

\begin{tabular}{|c|c|c|c|}
\hline Conditions $^{\text {a }}$ & $\mathbf{T A}^{\mathbf{c}}$ & $\%$ PI stained cells ${ }^{d}$ & CFUs ${ }^{\mathrm{e}}$ \\
\hline- & $\zeta^{-} \varepsilon^{(+)}$ & $2.6(1250)$ & $2.3 \times 10^{8}$ \\
\hline+ IPTG $^{\mathrm{b}}$ & $\zeta^{+} \varepsilon^{(+)}$ & $30(1314)$ & $3.8 \times 10^{3}$ \\
\hline$+\mathrm{IPTG}+\mathrm{Xyl}^{\mathrm{b}}$ & $\zeta^{+} \varepsilon^{+}$ & $9.8(1642)$ & $2.2 \times 10^{7}$ \\
\hline+ Fos & $\zeta^{-} \varepsilon^{(+)}$ & $73(1055)$ & $6.7 \times 10^{5}$ \\
\hline+ Fos + Xyl ${ }^{b}$ & $\zeta^{-} \varepsilon^{+}$ & $70(1432)$ & $6.6 \times 10^{5}$ \\
\hline+ IPTG $^{\mathrm{b}}+$ Fos & $\zeta^{+} \varepsilon^{(+)}$ & $75(1247)$ & $<1.0 \times 10^{2}$ \\
\hline$+\mathrm{IPTG}+\mathrm{Fos}+\mathrm{Xyl}^{\mathrm{b}}$ & $\zeta^{+} \varepsilon^{+}$ & $68(1560)$ & $2.3 \times 10^{5}$ \\
\hline
\end{tabular}

${ }^{a}$ BG1125 $\left(\zeta^{+}\right)$cells bearing pCB799-borne $\varepsilon$ gene were exponentially grown to $\sim 5 \times 10^{7}$ cells $/ \mathrm{mL}$ in MMS7 containing $0.005 \% \mathrm{Xyl}$ (denoted as $\left.\varepsilon^{(+)}\right)$; ${ }^{\mathrm{b}}$ Then, IPTG $(1 \mathrm{mM}$ to express $\zeta$ toxin) or Fos $(2 \times$ MIC) or both agents simultaneously were added and the cells incubated for $120 \mathrm{~min}$. In the conditions where Xyl $(0.5 \%)$ was added at min 120 (to express wt $\varepsilon_{2}$ antitoxin, denoted as $\varepsilon^{+}$), the cells were further incubated for $30 \mathrm{~min}$ and CFUs were counted on LB plates containing also Xyl; ${ }^{c}$ The presence or the absence of induction of $\zeta$ or $\varepsilon$ gene is indicated with a + or - , respectively, and the $(+)$ symbol denotes the low level of expression of the $\varepsilon$ gene by addition of $0.005 \%$ Xyl necessary to titrate basal expression of the wt $\zeta$ toxin; ${ }^{d}$ The percentage of cells stained with PI are indicated and number of cells analyzed are shown in parentheses; ${ }^{\mathrm{e}} \mathrm{CFUs} / \mathrm{mL}$ were measured by plating appropriate dilutions on LB or LB-Xyl ( $0.5 \%$ final concentration) plates. The results are the average of at least four independent experiments and are within a $10 \%$ standard error.

\section{Experimental Section}

\subsection{Bacterial Strains, Media and Growth Conditions}

The bacterial strains BG689 and BG1125 bearing pCB799 used in this study were previously reported [12] and are described in Table 1. Addition of low Xyl concentration (0.005\%) to BG1125 bearing pCB799 allowed the synthesis of low but significant $\varepsilon_{2}$ antitoxin levels, expressed from pCB799-borne $\varepsilon$ gene, necessary to titrate the basal $\zeta$ toxin levels and to avoid genetic rearrangements [14]. BG1125 cells bearing pCB799-borne $\varepsilon$ gene were grown in the presence of low levels of Xyl $(0.005 \%)$ necessary to induce a basal expression of the $\varepsilon_{2}$ antitoxin. Low levels of the $\varepsilon_{2}$ antitoxin are necessary to titrate the $\sim 40 \zeta$ toxin monomers/CFUs that are produced from escapes of 
LacI repression in the BG1125 strain. Addition of $1 \mathrm{mM}$ IPTG, increased $\zeta$ toxin to a plateau concentration of $\sim 1,700 \mathrm{wt} \zeta$ monomers/CFU at $\sim 30 \mathrm{~min}$, and the steady-state level of $\zeta$ remained for at least $240 \mathrm{~min}$ [see 14,15]. This toxin concentration is comparable to the level of wt $\zeta$ toxin, when the gene is in its native context and transcribed from its native promoter (cells bearing pBT233-borne $\omega \varepsilon \zeta$ operon that are neutralized by saturating $\varepsilon_{2}$ antitoxin concentrations) [11].

Expression of the xylR $P_{\text {xylA }} \zeta Y 83 \mathrm{C}$ cat cassette (BG689 strain) was induced by addition of $0.5 \% \mathrm{Xyl}$ and expression of the lacI $P_{h s p} \zeta s p c$ cassette (BG1125 strain) by addition of $1 \mathrm{mM}$ IPTG. The BG689 or BG1125 bearing pCB799 isogenic strains were grown to mid-exponential phase $\left(\sim 5 \times 10^{7}\right.$ cells $\left./ \mathrm{mL}\right)$ at $37{ }^{\circ} \mathrm{C}$ in MMS7 supplemented with the required amino acid (methionine and tryptophan) at $50 \mu \mathrm{g} / \mathrm{mL}[12]$.

The antimicrobial, except Van, used a 4-times MIC, was added at twice MIC. Cells were taken after $120 \mathrm{~min}$, washed with fresh pre-warmed media (to remove the inducer and/or antimicrobial) and appropriated dilutions were plated on LB agar plates containing glucose (which switches off $\zeta$ Y 83C expression) or on LB agar plates containing or not Xyl to express the $\varepsilon_{2}$ antitoxin ( $\zeta$ toxin cassette). The survival rate, derived from the number of CFUs obtained in a given condition relative to the CFUs of the non-induced/non-antimicrobial treated control is documented.

The MIC of the different antimicrobials tested was estimated by exposing $1-3 \times 10^{6}$ cells $/ \mathrm{mL}$ for $16 \mathrm{~h}$ at $37{ }^{\circ} \mathrm{C}$ in MMS7 with shaking $(240 \mathrm{rpm})$ to different concentrations of the antimicrobials employed, and is the mean of the minimum concentration that gave no growth in three independent experiments. In the absence of inducer, the presence of the $\zeta$ Y83C (BG689 strain) or $\zeta$ gene and pCB799-borne $\varepsilon$ gene (BG1125) does not affect the MIC [15].

For $\zeta$ toxin purification the stop codon of the $\zeta$ gene was replaced by 6 His and a stop codon. This $\zeta$ variant shows the same phenotype that wt $\zeta$ when expressed in B. subtilis cells [16]. This $\zeta$ gene variant was non-clonable in $E$. coli cells under the transcriptional control of the T7 RNA polymerase-dependent promoter $\left(P_{\mathrm{T} 7}\right)$, but cloning of the $\omega$ and $\varepsilon$ genes, under its native RNA polymerase $\sigma^{\mathrm{A}}$-dependent promoter $\left(P_{\omega}\right)$, in opposite orientation, overcame the toxicity exerted by the $\zeta$ variant, leading to pCB920 (Table 1).

\subsection{Fluorescence Microscopy}

To determine the proportion of "membrane-compromised" cells, they were harvested by centrifugation, washed to remove toxin inducer and/or the antimicrobial, and were stained with SYTO 9, which stains all bacteria with green fluorescence, and PI, which stains membrane-compromised bacteria with red fluorescence, according to the manufacturer's instructions (Molecular Probes, Leiden). Cells were visualized using a BX61 Olympus microscope and Olympus CCD DP70 camera, with the appropriate filters as described [12].

\subsection{Protein Purification}

Plasmid pCB920-borne $\zeta$, under the control of promoter 10 of phage T7 $\left(P_{10}\right)$, and $\varepsilon$ gene under the control of native $P_{\omega}$, was used to overexpress $\zeta$ in E. coli BL21(DE3) cells harboring pCB920. IPTG was added to induce the expression of T7 RNAP (lacI lac $c_{\mathrm{Uv}} \mathrm{T} 7$ gene 1), and 30 min later rifampicin was added to selectively block the expression of the $\omega$ and $\varepsilon$ genes. After 120 min of 
$\zeta$ expression from rifampicin-resistant $P_{10}$, and full decay of the $\varepsilon_{2}$ antitoxin, the cells were harvested. The over-expressed long-living $\zeta$ toxin was purified in two steps as follows: cells were lysed using the French Press in buffer A (50 mM phosphate buffer pH 7.5, $100 \mathrm{mM} \mathrm{NaCl}, 5 \%$ glycerol), and soluble $\zeta$ protein was bound to a Ni-NTA column, and eluted using an imidazole gradient ( 2 to $75 \mathrm{mM}$ ). The fractions containing the $\zeta$ protein were diluted to $25 \mathrm{mM} \mathrm{NaCl}$, and loaded in a Q Sepharose column. Protein $\zeta$ was eluted in buffer B $(50 \mathrm{mM}$ Tris- $\mathrm{HCl} \mathrm{pH} 7.5,5 \%$ glycerol $)$ containing a gradient of $\mathrm{NaCl}$ (25 to $150 \mathrm{mM})$. The fractions containing the $\zeta$ protein were dialyzed against buffer $\mathrm{C}(50 \mathrm{mM}$ Tris- $\mathrm{HCl} \mathrm{pH} 7.5,100 \mathrm{mM} \mathrm{NaCl}$ ) containing $50 \%$ glycerol and stored at $-20{ }^{\circ} \mathrm{C}$.

\subsection{Biochemical Assays}

The ATPase or GTPase activity of $\zeta$ was measured by incubating increasing ATP or GTP concentrations (containing $\left.10 \mathrm{nM}\left[\alpha^{-32} \mathrm{P}\right]-\mathrm{ATP}\right)$ and a fixed amount of UNAG $(2 \mathrm{mM})$ with purified toxin in buffer A (50 mM Tris-HCl pH 7.5; $\left.50 \mathrm{mM} \mathrm{NaCl} ; 1 \mathrm{mM} \mathrm{MgCl}_{2}\right)$ at $30{ }^{\circ} \mathrm{C}$. Then, TLCs of the radiolabeled nucleotides or sugar nucleotides were performed on polyethyleneimine-cellulose plates with $0.85 \mathrm{M} \mathrm{KH}_{2} \mathrm{PO}_{4}$ (pH 3.4) as the mobile phase as described [14] followed by autoradiography.

Quantitative production of UNAG-3P, degradation of ATP and accumulation of ADP was determined by Maldi-TOF analysis as described [32]. ATP and UNAG were incubated or not with $\zeta$ toxin in buffer B (10 mM Tris-HCl, $\left.\mathrm{pH} 7.5,50 \mathrm{mM} \mathrm{NaCl}, 1 \mathrm{mM} \mathrm{MgCl}_{2}\right)$ for $30 \mathrm{~min}$. Equal volume of the analyte solution $(1 \mu \mathrm{L})$ was mixed with $1 \mathrm{~mL}$ of matrix solution $(2,4,6$ trihydroxyacetophenone or 2,3,6-trihydroxyacetophenone, $10 \mathrm{mg} / \mathrm{mL}$ in acetonitrile/water, 1:1 v/v) and $1 \mathrm{~mL}$ of ammonium citrate $(50 \mathrm{mg} / \mathrm{mL}$ in water). Then $1 \mathrm{~mL}$ of the mixture was applied to the MALDI-TOF sample plate and air dried. The mass spectra were acquired using a Ultraflex III TOFTOF (BRUKER) mass spectrometer (equipped with a ND:YAG laser) operating in negative reflector mode.

\section{Conclusions}

Toxin $\zeta$-mediated dormancy induction can be attributed to the reduction of the ATP and/or GTP pools, the increment of the (p) ppGpp pool size and the accumulation of unreactive UNAG-3P among other changes (this work) [9]. We report here that purified $\zeta$ is mainly an UNAG-dependent ATPase rather than a GTPase. Preliminary data suggested that ATP hydrolysis and accumulation of unreactive UNAG-3P are not strictly coupled reactions. Our results indicate that physiological $\zeta$ toxin concentrations enhance the efficacy of antimicrobials that inhibit cell wall biosynthesis (Figure 3 ). A similar $\zeta$-mediated sensitization to other three different classes of antimicrobials was reported previously [15].

Our results are incompatible with the hypothesis that $\zeta$ or PezT depletes the UNAG pool to generate an irreversible positive feedback loop, and as the cell wall blockage takes effect, cell wall weakening should instigate a suicide program and autolysis [9]. We surmise that cells can readjust their metabolism during situations where $\zeta$ toxin activation is temporary and reversible. Our in vivo experiments suggest that transient $\zeta$ toxin expression (120 min) reversibly induces a dormant state, leads to a sub-fraction $(25 \%-35 \%)$ of the population stained with PI, and a minor subpopulation of cells exhibits non-inheritable toxin tolerance. Similarly, addition of Fos triggers stasis, leads to a fraction (65\%-75\%) of PI stained cells (suggesting cell death), and a minor subpopulation of cells are 
stochastically tolerant to Fos action. After $\zeta$ toxin expression and Fos addition, subsequent expression of the $\varepsilon_{2}$ antitoxin specifically reverses $\zeta$-induced dormancy, but it fails to reduce the proportion of Fos-induced PI stained cells.

From the results obtained in this work and previous results, we propose that the modus operandi of $\zeta$ toxin is to induce diverse responses to cope with stress, and reduction of the UNAG pool is one among them, rather than instigating a suicide program by depleting UNAG. A similar scenario is observed upon long time $\zeta$ toxin accumulation (360 or $480 \mathrm{~min}$ ). Here, tolerant cells might enter into the viable-but-nonculturable state, but massive cell lysis was not observed. Conversely, after $60 \mathrm{~min}$ over-expression in E. coli cells, the PezT $\Delta \mathrm{C} 242$ toxin blocks cell wall synthesis and triggers massive autolysis with few intact cells with an ovoid morphology [9], but such massive autolysis was not observed upon $\zeta$ toxin over-expression [31]. It is likely that the truncated and attenuated version of the PezT toxin (PezT $\Delta \mathrm{C} 242)$ is bactericidal rather than bacteriostatic in nature.

\section{Acknowledgments}

The research was partially financed by the Ministerio de Economía y Competividad (MINECO) BFU2012-39879-C02-01 to J.C.A., by the MINECO (BFU2012-39879-C02-02) and by the Comunidad de Madrid (CM-BIO0260-2006) to S.A. MT is a $\mathrm{PhD}$ fellow of the International Fellowship Programme of La Caixa Foundation (La Caixa/CNB). We thank Maite Alonso Pascual for mass spectroscopy determinations.

\section{Author Contributions}

Mariangela Tabone, Silvia Ayora and Juan C. Alonso conceived and designed the experiments; Mariangela Tabone and Silvia Ayora performed the experiments; Mariangela Tabone, Silvia Ayora and Juan C. Alonso analyzed the data; Mariangela Tabone contributed reagents/materials/analysis tools; Silvia Ayora and Juan C. Alonso wrote the paper.

\section{Conflicts of Interest}

The authors declared no conflict of interest.

\section{References}

1. Engelberg-Kulka, H.; Glaser, G. Addiction modules and programmed cell death and antideath in bacterial cultures. Annu. Rev. Microbiol. 1999, 53, 43-70.

2. Gerdes, K.; Christensen, S.K.; Lobner-Olesen, A. Prokaryotic toxin-antitoxin stress response loci. Nat. Rev. Microbiol. 2005, 3, 371-382.

3. Van Melderen, L.; Saavedra De Bast, M. Bacterial toxin-antitoxin systems: More than selfish entities? PLoS Genet. 2009, 5, e1000437.

4. Leplae, R.; Geeraerts, D.; Hallez, R.; Guglielmini, J.; Dreze, P.; Van Melderen, L. Diversity of bacterial type II toxin-antitoxin systems: A comprehensive search and functional analysis of novel families. Nucleic Acids Res. 2011, 39, 5513-5525. 
5. Alonso, J.C.; Balsa, D.; Cherny, I.; Christensen, S.K.; Espinosa, S.; Francuski, D.; Gazit, E.; Gerdes, K.; Hitchin, E.; Martín, M.T.; et al. Bacterial toxin-antitoxin systems as targets for the development of novel antibiotics. In Enzyme-Mediated Resistance to Antibiotics; Bonomo, R.A., Tolmasky, M., Eds.; American Society for Microbiology Press: Washington, DC, USA, 2007; pp. 313-329.

6. Mutschler, H.; Meinhart, A. $\varepsilon / \zeta$ systems: Their role in resistance, virulence, and their potential for antibiotic development. J. Mol. Med. 2011, 89, 1183-1194.

7. Meinhart, A.; Alonso, J.C.; Strater, N.; Saenger, W. Crystal structure of the plasmid maintenance system $\varepsilon / \zeta$ : Functional mechanism of toxin $\zeta$ and inactivation by $\varepsilon_{2} \zeta_{2}$ complex formation. Proc. Natl. Acad. Sci. USA 2003, 100, 1661-1666.

8. Khoo, S.K.; Loll, B.; Chan, W.T.; Shoeman, R.L.; Ngoo, L.; Yeo, C.C.; Meinhart, A. Molecular and structural characterization of the PezAT chromosomal toxin-antitoxin system of the human pathogen Streptococcus pneumoniae. J. Biol. Chem. 2007, 282, 19606-19618.

9. Mutschler, H.; Gebhardt, M.; Shoeman, R.L.; Meinhart, A. A novel mechanism of programmed cell death in bacteria by toxin-antitoxin systems corrupts peptidoglycan synthesis. PLoS Biol. 2011, 9, e1001033.

10. Meinhart, A.; Alings, C.; Strater, N.; Camacho, A.G.; Alonso, J.C.; Saenger, W. Crystallization and preliminary $\mathrm{x}$-ray diffraction studies of the $\varepsilon \zeta$ addiction system encoded by Streptococcus pyogenes plasmid pSM19035. Acta Crystallogr. Sect. D: Biol. Crystallogr. 2001, 57, 745-747.

11. Camacho, A.G.; Misselwitz, R.; Behlke, J.; Ayora, S.; Welfle, K.; Meinhart, A.; Lara, B.; Saenger, W.; Welfle, H.; Alonso, J.C. In vitro and in vivo stability of the $\varepsilon_{2} \zeta_{2}$ protein complex of the broad host-range Streptococcus pyogenes pSM19035 addiction system. Biol. Chem. 2002, 383, 1701-1713.

12. Lioy, V.S.; Martin, M.T.; Camacho, A.G.; Lurz, R.; Antelmann, H.; Hecker, M.; Hitchin, E.; Ridge, Y.; Wells, J.M.; Alonso, J.C. pSM19035-encoded $\zeta$ toxin induces stasis followed by death in a subpopulation of cells. Microbiology 2006, 152, 2365-2379.

13. Brzozowska, I.; Zielenkiewicz, U. The ClpXP protease is responsible for the degradation of the $\varepsilon$ antidote to the $\zeta$ toxin of the streptococcal psm19035 plasmid. J. Biol. Chem. 2014, 289, 7514-7523.

14. Lioy, V.S.; Machon, C.; Tabone, M.; Gonzalez-Pastor, J.E.; Daugelavicius, R.; Ayora, S.; Alonso, J.C. The $\zeta$ toxin induces a set of protective responses and dormancy. PLoS One 2012, 7, e30282.

15. Tabone, M.; Lioy, V.S.; Ayora, S.; Machon, C.; Alonso, J.C. Role of toxin $\zeta$ and starvation responses in the sensitivity to antimicrobials. PLoS One 2014, 9, e86615.

16. Lioy, V.S.; Rey, O.; Balsa, D.; Pellicer, T.; Alonso, J.C. A toxin-antitoxin module as a target for antimicrobial development. Plasmid 2010, 63, 31-39.

17. Cardenas, P.P.; Carzaniga, T.; Zangrossi, S.; Briani, F.; Garcia-Tirado, E.; Deho, G.; Alonso, J.C. Polynucleotide phosphorylase exonuclease and polymerase activities on single-stranded DNA ends are modulated by RecN, SsbA and RecA proteins. Nucleic Acids Res. 2011, 39, 9250-9261. 
18. Du, W.; Brown, J.R.; Sylvester, D.R.; Huang, J.; Chalker, A.F.; So, C.Y.; Holmes, D.J.; Payne, D.J.; Wallis, N.G. Two active forms of UDP- $N$-acetylglucosamine enolpyruvyl transferase in Gram-positive bacteria. J. Bacteriol. 2000, 182, 4146-4152.

19. Marquardt, J.L.; Brown, E.D.; Lane, W.S.; Haley, T.M.; Ichikawa, Y.; Wong, C.H.; Walsh, C.T. Kinetics, stoichiometry, and identification of the reactive thiolate in the inactivation of UDP-GlcNac enolpyruvoyl transferase by the antibiotic fosfomycin. Biochemistry 1994, 33, 10646-10651.

20. Baum, E.Z.; Montenegro, D.A.; Licata, L.; Turchi, I.; Webb, G.C.; Foleno, B.D.; Bush, K. Identification and characterization of new inhibitors of the Escherichia coli MurA enzyme. Antimicrob. Agents Chemother. 2001, 45, 3182-3188.

21. Breukink, E.; de Kruijff, B. Lipid II as a target for antibiotics. Nat. Rev. Drug Discov. 2006, 5, 321-332.

22. Archibald, A.R.; Hancock, I.C.; Harwood, C.R. Cell wall structure, synthesis, and turnover. In Bacillus subtilis and other Gram-Positive Bacteria; Sonenshein, A.L., Hoch, J.A., Losick, R., Eds.; American Society for Microbiology Press: Washington, DC, USA, 1993; pp. 381-410.

23. Foster, S.J.; Popham, D.L. Structure and synthesis of cell wall, spore cortex, teichoic acids, S-layers, and capsules. In Bacillus subtilis and Its Closest Relatives; Sonenshein, A.L., Hoch, J.A., Losick, R., Eds.; American Society for Microbiology Press: Washington, DC, USA, 2002; pp. 21-41.

24. Bhavsar, A.P.; Brown, E.D. Cell wall assembly in Bacillus subtilis: How spirals and spaces challenge paradigms. Mol. Microbiol. 2006, 60, 1077-1090.

25. Reynolds, P.E. Structure, biochemistry and mechanism of action of glycopeptide antibiotics. Eur. J. Clin. Microbiol. Infect. Dis. 1989, 8, 943-950.

26. Wright, A.J. The penicillins. Mayo Clin. Proc. 1999, 74, 290-307.

27. Jordan, S.; Hutchings, M.I.; Mascher, T. Cell envelope stress response in Gram-positive bacteria. FEMS Microbiol. Rev. 2008, 32, 107-146.

28. Bigger, J.W. Treatment of staphylococcal infections with penicillin. Lancet 1944, 244, 497-500.

29. Gefen, O.; Balaban, N.Q. The importance of being persistent: Heterogeneity of bacterial populations under antibiotic stress. FEMS Microbiol. Rev. 2009, 33, 704-717.

30. Lewis, K. Persister cells. Annu. Rev. Microbiol. 2010, 64, 357-372.

31. Zielenkiewicz, U.; Ceglowski, P. The toxin-antitoxin system of the streptococcal plasmid pSM19035. J. Bacteriol 2005, 187, 6094-6105.

32. Gunther Sillero, M.A.; Perez-Zuniga, F.; Gomes, J.; de Carvalho, A.I.; Martins, S.; Silles, E.; Sillero, A. Synthesis of FUDP- $N$-acetylglucosamine and FUDP-glucose in Saccharomyces cerevisiae cells treated with 5-fluorouracil. FEMS Yeast Res. 2008, 8, 257-265.

(C) 2014 by the authors; licensee MDPI, Basel, Switzerland. This article is an open access article distributed under the terms and conditions of the Creative Commons Attribution license (http://creativecommons.org/licenses/by/3.0/). 